International Journal of Advances in Materials Science and Engineering (IJAMSE) Vol.4, No.3, July 2015

\title{
Developing Surface Metal Matrix Composites: A Comparative Survey
}

\author{
B. Ratna Sunil \\ Department of Mechanical Engineering, \\ Rajiv Gandhi University of Knowledge Technologies (AP-IIIT), Nuzvid 521202, India
}

\begin{abstract}
Surface metal matrix composites (MMCs) are a class of engineered materials which contain secondary phase that is distributed at the surface and no composition change is found at the core. Especially for applications where surface requires more hardness and wear resistance, surface composites play a promising role. There are many routes such as laser melt treatment, vapour deposition, centrifugal casting, plasma spraying which are used to produce surface composites and reported in the literature. Solid state processing methods for example friction assisted techniques are the other recent developments have grabbed a great interest in developing surface composites. The aim of the present review is to summarize different processing routes which have been developed to produce surface MMCs. Emphasis has been given to discuss the mechanism behind the formation of composite during processing and to compare the advantages and limitations of these processes.
\end{abstract}

\section{KEYWORDS}

Surface composites, MMCs, liquid state methods, solid state methods

\section{INTRODUCTION}

Metals, ceramics and polymers are the three basic groups of materials. The combination of two or more than two materials belong to these basic groups gives composites. Among the composites, metal matrix composites (MMCs) are a promising category evolved as a class of modern engineered materials and demonstrated their promising behaviour as best suited for wide variety of applications including aerospace, automobile and marine industries.

In MMCs, matrix material is a metal in the form of pure phase or alloy. Usually the dispersing phase is hard ceramic or metallic compounds in the form of fine or coarse particles or fibres. The matrix material exhibits soft nature compared with the dispersing hard phase. Aluminium, copper, magnesium and titanium are a few examples of matrix materials and $\mathrm{SiC}, \mathrm{Al}_{2} \mathrm{O}_{3}, \mathrm{TiB}_{2}$, WC, CNTs are a few examples for reinforcing secondary phase materials [1]. The advantages of MMCs include high stiffness, high dimensional stability with high strength and high elastic modulus, high electrical and thermal conductivity compared with other composites. Other important properties of MMCs are high wear resistance and fatigue resistance and good creep properties compared with that of base material [2]. But higher manufacturing cost and difficult to machining are the major limitations with MMCs [3]. However, the recent advancements in the manufacturing industry have enabled production engineers to address these limitations to make MMCs more feasible to use in different applications. Components in aircrafts, satellites, jet engines and missiles in aerospace industry; engine components, piston and break disks in automobile industry are a few promising applications of MMCs [4]. 


\section{Surface Metal Matrix Composites}

It has been well understood that in some applications the surface properties are crucial and dictate the performance and life span of a component. In some components, surface with high hardness and wear resistance, at the same time ductile core with high toughness may be required; for example in machine tools where vibrations or fluctuating loads are a common observation. For such applications, composition of the surface of the component can be changed by introducing secondary phase at the surface up to certain thickness while the composition of the core of the component is unchanged. Therefore, considering its mechanical properties, the surface of the component exhibits higher hardness and wear resistance and the material in bulk experience negligible loss to its toughness. Similarly, chemical properties also are changed at the surface compared with inner core. Such material behaves like a composite at the surface and without affecting the properties at the core and can be called as surface MMCs as schematically shown in Fig 1.

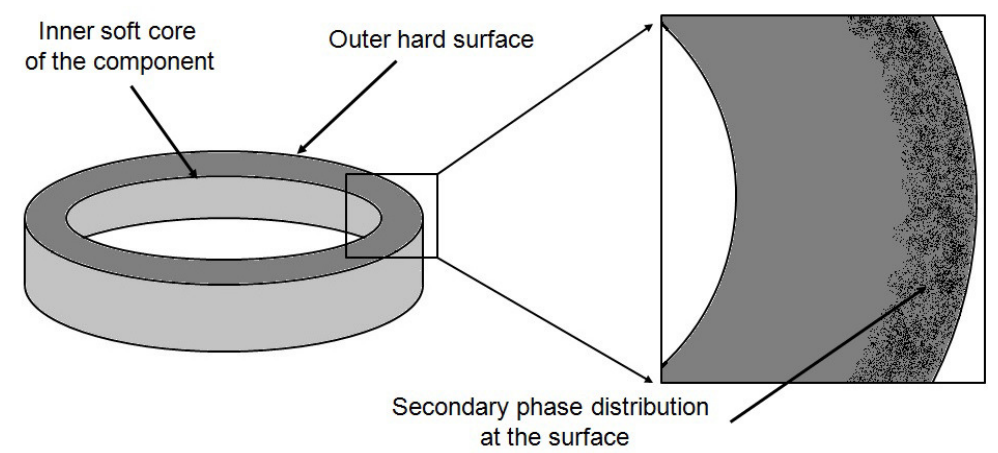

Fig 1 Schematic representation of a component showing hard composite at the surface with soft inner core.

\section{Manufacturing Processes of Surface MMCs}

Engineering the surface by introducing secondary phase to produce surface MMCs requires special category methods which can modify only the surface up to certain depth without affecting the inner core of the material. We can classify these methods as liquid state and solid state methods based on the state of the matrix (liquid or solid) during processing. In liquid state methods, matrix material is melted whereas in solid state methods, composite fabrication is done without melting the matrix material. Laser melt treatment, vapour deposition, centrifugal casting, plasma spraying are the examples of liquid state methods. Friction surfacing and friction stir processing are the examples for solid state methods.

\subsection{Liquid state methods}

Liquid state methods involve the matrix material transformation from solid to liquid or vapour state during process. Then the secondary phase is introduced into the molten matrix and surface composites are produced.

\subsubsection{Laser melt treatment}

In laser melt treatment, electromagnetic radiation in the form of photons (laser beam) is used to melt the matrix material as the secondary phase particles are supplied through a nozzle by a carrier inert gas [5]. Fig 2 shows the schematic representation of laser melt treatment process. The 
thickness of the surface which is melted during laser melt treatment depends on the energy density of the laser beam and type of the work piece material. The mechanism involves melting the surface and dispersing the secondary phase particles when the surface is in molten state and form a surface composite after cooling. There is a possibility of reaction between secondary phase and the matrix material as the temperature of the molten metal is very high. Therefore, other intermediate phases can form at the particle matrix interfaces. The phase stability of the secondary phase particles at the elevated temperature during the process also need to be considered in designing the surface composites using laser melt treatment. Since the solidification happens at the surface level, critical control over the processing parameters is required to achieve optimum microstructure at the surface layer [6].

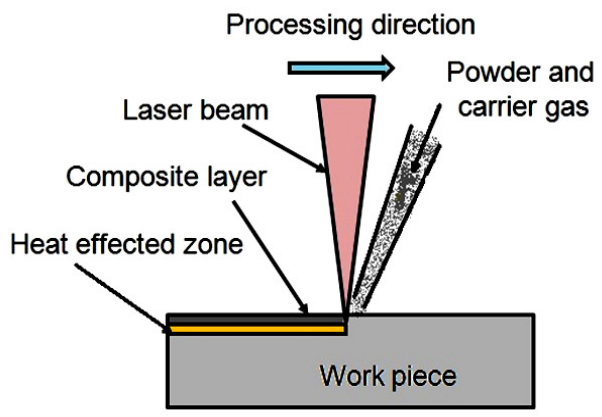

Fig 2 Schematic representation of fabricating surface MMCs by laser melt technique

\subsubsection{Vapour deposition methods}

The mechanism in vapour deposition processes is simple. The coating material is transferred to the surface of the work piece molecule by molecule or atom by atom. There are two basic types of vapour deposition methods named as chemical vapour deposition (CVD) and physical vapour deposition (PVD). In CVD method, a precursor gas or combination of gases flow over a heated work piece kept in a chamber. Due to the chemical reaction at the surface or some times on the surface, formation of a thin phase is resulted as shown in Fig 3 (a). Composite layers can be produced by supplying corresponding gases through the chamber [7, 8]. In PVD method, the material which is going to be coated on the work piece is evaporated with the help of high energy sources such as electron beam or sputtering by magnetrons and allowed to deposit on the work piece kept in vacuum or a protective atmosphere as shown in Fig 3 (b). Reactive gases can be flushed into the chamber which can form chemical bonds with the materials present in the chamber in vapour state and deposit on the surface of the work piece as different compounds $[9,10,11]$.

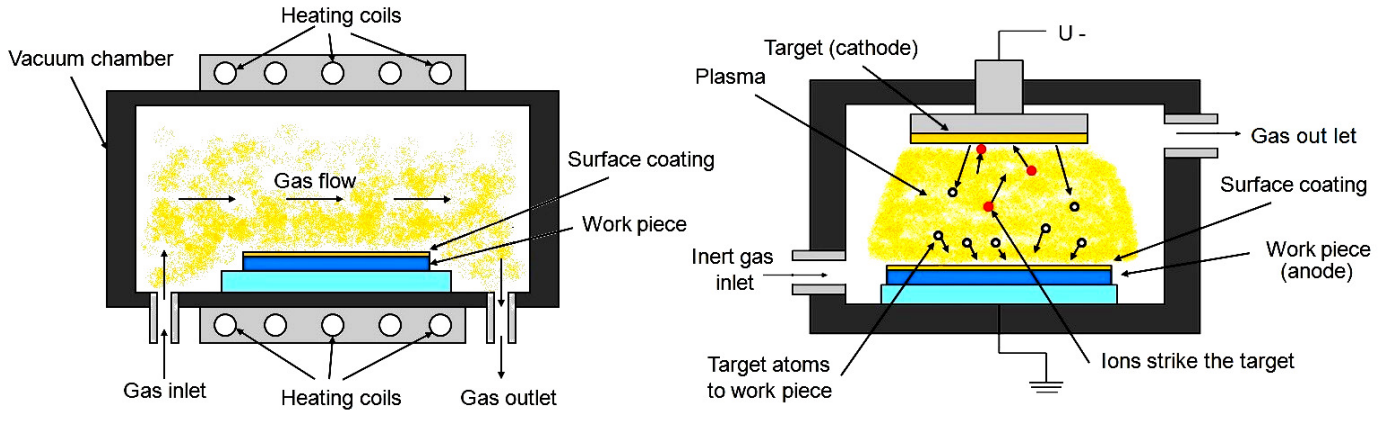

(a)

(b)

Fig 3 Schematic representation of vapour deposition methods used to fabricate surface MMCs: a) CVD method and b) PVD method 


\subsubsection{Centrifugal casting}

This process is best suited for producing axisymmetric objects. In centrifugal casting, the secondary phase particles are added to the molten metal and poured into a rotating hot mould as schematically shown in Fig 3. Due to the centrifugal force, these secondary phase particles are pushed in the radial direction to the circumference of the mould. The difference in densities of matrix material and the dispersing phase play an important role in distributing the secondary phase at the surface of the cast component. Geometry of the object is restricted in centrifugal casting which is a limitation. Only axisymmetric objects can be produced by centrifugal casting [12]

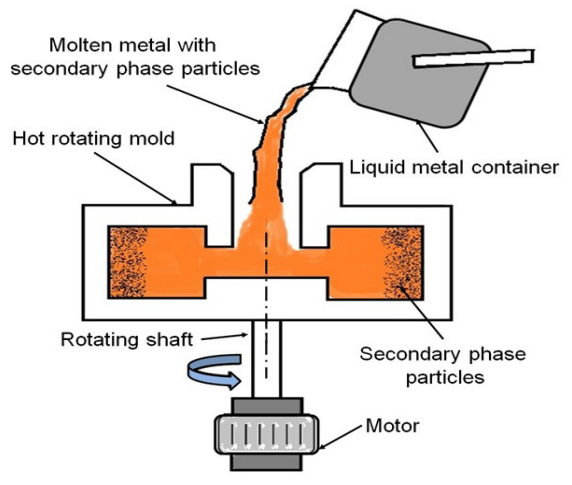

Fig 4 Schematic representation of centrifugal casting used to produce surface MMCs

\subsubsection{Plasma spraying}

When a gas passes through high temperature zone, the molecules split into atoms and further into electrons and ions. This ionized hot gas containing electrons and ions is called as plasma. Very high temperatures can be attained in plasma up to a few thousands degree Celsius. Plasma can be used to melt the material to do welding or cutting. Another important application of plasma is surface coatings. There are two modes of plasma generation known as transferred and nontransferred. In transferred mode, work piece is a part of the circuit and plasma is stuck between the cathode and work piece. In, non-transferred plasma mode, work piece is not a part of the circuit and the nozzle acts as anode. Plasma is generated between cathode and nozzle and is pushed by the supplying plasma gas to the work piece. Transferred mode is used for welding and cutting operations whereas non-transferred mode is used for surface coatings. Plasma spraying is an advanced surface coating process which can be used to develop surface composites [13].

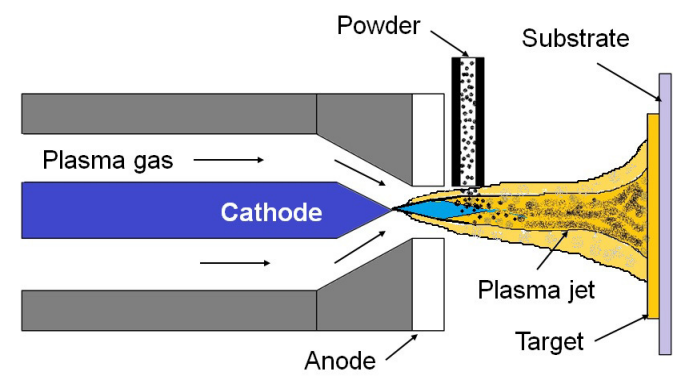

Fig 5 Schematic representation of plasma spraying process 
In plasma spraying, the materials intended to be formed as a composite coating are introduced into the plasma jet at the entrance of the nozzle as shown in Fig 5. The material can be introduced in the form of powder or a continuous rod. The feed material is melted due to the high temperature in the plasma jet and forced towards the substrate as liquid droplets. These molten droplets rapidly solidify during the deposition on the substrate and form lamellar coatings. If the feed material is a combination of different phases, the substrate is coated with a composite layer.

\subsection{Solid state methods}

Solid state processing techniques do not result transformation of the matrix material to liquid phase during the process. Secondary phase is introduced into the matrix material in solid state itself and offer relatively many advantages over conventional liquid phase processing techniques.

\subsubsection{Friction surfacing}

Friction surfacing is one of the friction assisted processes in which a consumable rod known as mechtrode is used to deposit material on the surface of the work piece [14]. Fig 6 shows the schematic representation of friction surfacing process. A rotating consumable metallic rod is pressed against the work piece by applying a suitable axial load. Due to the friction between the work piece and the mechtrode, heat is generated which makes the mechtrode soft and the plasticized material is deposited from the mechtrode on the surface of the work piece due to the plastic deformation. As the consumable tool is travelling on the surface of the work piece, a layer is formed at the expense of the rotating mechtrode. Surface composites can be produced by using machtrode having different phases of desired composition. In developing surface composites, mechtrode is made of a composite material which is going to coat on the surface of the work piece. A strong metallurgical bond is established between the composite layer and the substrate and results surface composite.

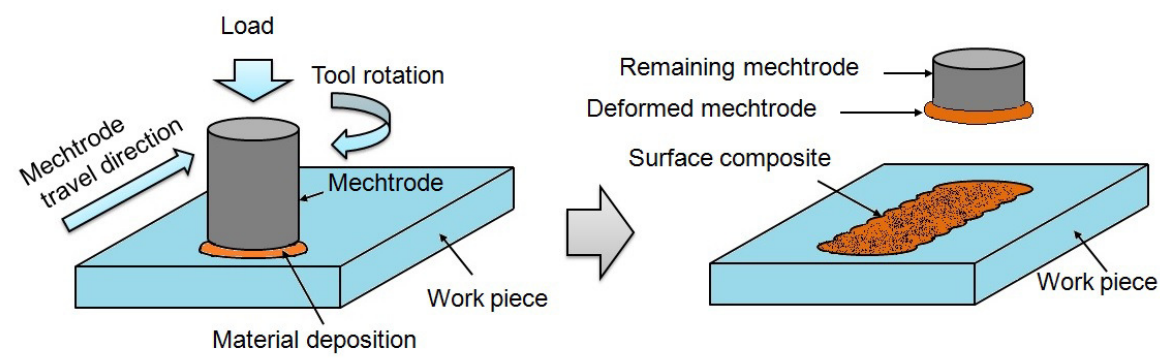

Fig 6 Schematic representation of friction surfacing to develop surface composites

\subsubsection{Friction stir processing}

Friction stir processing (FSP) is another potential technique among the friction assisted processes. FSP is a solid-state processing technique that has been developed from the basic principles of FSW and emerged as a promising tool to alter the surface microstructure of metallic sheets and plates [15]. In, FSW, a non-consumable rotating tool which has a pin at the end is inserted into the edges of the sheets or plates which are going to be joined and the rotating tool is plunged in traverse direction by applying a suitable load. The principle behind the joint formation and grain refinement in FSW has been explained by Mishra et al., $[15,16]$. The material flow in the solid state itself leads to form a solid-state joint and no part of the work piece undergoes melting in FSW [15]. The basic functions of the non-consumable tool in FSW are i) generating heat, ii) 
causing the movement of the material surrounding the pin and iii) holding the metal beneath the shoulder by providing a hydrostatic pressure.

During FSP, heat is generated within the sheet or plate by friction between the work piece and rotating tool pin and shoulder and by plastic deformation of the work piece material. The localized heating makes the material soft at the stirring zone and movement of the material is caused by the rotating tool pin. As the tool moves in forward direction, the hole that is formed by the tool traverse motion is filled with the material which moves from the front to rear of the tool. The material movement is suppressed to a level equal to the thickness of the sheet by the tool shoulder which continuously applies a suitable load throughout the process.

Fabrication of surface composites by FSP is another development in friction assisted processes in which the stirring action of the FSP tool is used to incorporate and distribute secondary phase in the matrix material. For the first time, Mishra et al., [17] demonstrated the fabrication of 5083Al$\mathrm{SiC}$ surface composite using FSP and reported in the literature. Usually a narrow groove or holes are produced on the surface of the sheet and the secondary phase particles are filled and FSP is carried out in order to produce the surface composite. The schematic representation of the process is shown in Fig 7.

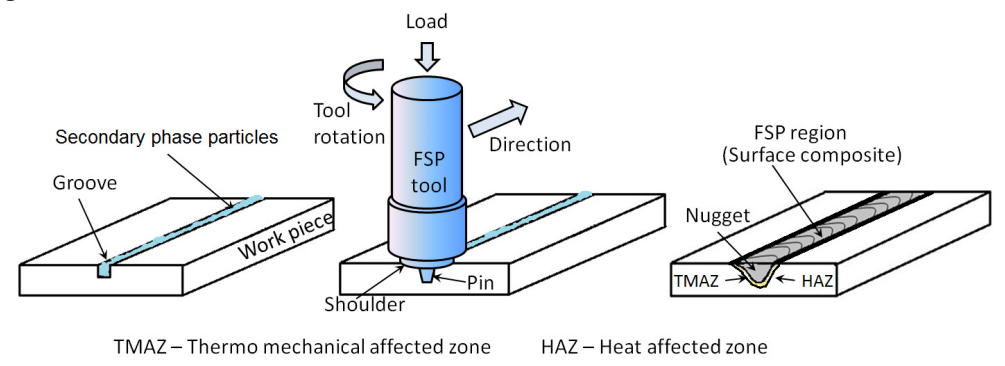

Fig 7 Schematic representation of friction stir processing (FSP) (Source: Ratna Sunil et al., [18])

The secondary phase is distributed within the stir zone during FSP due to the plastic flow of the material. The thickness of the surface composite layer is equal to the FSP tool pin length at the nugget zone. There are many factors which influence the successful formation of the composite in FSP. Tool geometry, processing parameters and type of work piece are important factors which dictate the success of the composite formation using FSP. The material flow during the FSP process involves several independent deformation processes such as extrusion and forging along with simultaneous localized heating and cooling [15].

\section{DISCUSSION}

Melting of matrix material is a common observation in all liquid state processing methods. There are a few issues such as possibility of oxidation and large grain size associated due to the phase transformation from solid to liquid. In laser melt treatment, knowing the response of the matrix material to the laser beam is important. Some metals reflect the laser beam so the generated heat is less and the effected surface thickness is also lower. Some materials absorb the energy and can be melted up to high depths. Therefore, the thickness of the surface composite layer depends on the response of the matrix material. The temperatures are also greater in laser melt treatment which affects the stability of the dispersing phase which is another problem.

Complex shaped components can be coated in developing surface composites using CVD process which is a great advantage. The thickness of the surface coating layer is uniform in CVD process. Deposition of secondary phases with very high purity is another advantage with CVD process and also CVD does not require high vacuum as it is require in PVD processes. Handling the 
precursors at room temperature is the main limitation with CVD processes. Similar to CVD process, wide variety of materials can be coated using PVD technologies. Surface composite produced from PVD are harder and more corrosion resistant. Most of the surface coatings exhibit excellent tribological properties. Surface composite layers produced by PVD are most environmental friendly. Requirement of high vacuum and special kind of equipment make these vapour deposition methods costly. Developing surface composites with higher thickness is another limitation by considering economical issues which is a major limitation in vapour deposition methods.

Centrifugal casting is another promising method in developing surface composites but, the geometry of the component is a great limitation. Components which are axisymmetric only can be produce from centrifugal casting. However, surface properties of the components produced from centrifugal casting are excellent which make this process is best suited for axisymmetric objects compared with other methods. Plasma spraying processing gives surface composites with excellent properties and the process can be called as high efficiency process. The mechanical properties of the surface layer produced by plasma spraying on the substrate are superior compared with the surface layers produced by other methods. However, the operating temperatures are very high in plasma spraying which may not be suitable for those phases having instability at such a high temperatures. Emission of higher amount of radiation is another limitation with plasma spraying methods.

Friction assisted processes such as friction surfacing and friction stir processing are recently developed techniques have offered great advantages in producing surface composites. Fabricating the composite in solid state itself is the significant advantage of friction assisted processes. In friction surfacing, there are many material combinations which can be used to produce mechtrode. However, preparing the secondary phase in the form of mechtrode which is coated on the surface of the work piece is the main limitation. In FSP, incorporating the secondary phase particles and grain size reduction at the surface can be achieved simultaneously which is an advantage with FSP. The problems associated with melting and solidification such as large grain size and stability of the dispersing secondary phase are completely eliminated in both the friction surfacing and FSP processes. However, difficulty is high in producing surface composites with the material systems which have high melting temperatures and show brittle nature. The surface geometry of work piece is another limitation with friction assisted processes. Curved surfaces are slight difficult to process compared with flat surfaces in friction assisted processes. Obtaining uniform dispersion throughout the stir zone is also another limitation and challenge in developing surface composites using FSP. Overall, FSP has a great potential to develop surface composites of wide variety of material systems which can be adapted in many industrial applications.

\section{CONCLUSION}

Producing surface composites which exhibit different properties at the surface compared with the core of the material without altering the composition at the core of the component has its own demand in many industrial applications. Wide variety of methods which fall under two basic routes known as liquid state and solid state processing techniques have been developed to produce surface MMCs. Each method has its own advantages and limitations from the processing and work material perspectives. Friction assisted processes are newly evolving solid state methods which have a great potential compared with other processes in developing surface MMCs but need further developments to address the issues concerning the distribution of the secondary phase. Component geometry, required thickness of the composite at the surface, purity of the composite layer and stability of the secondary phase are the main factors need to be considered while adapting a specific method in developing surface MMCs. 
International Journal of Advances in Materials Science and Engineering (IJAMSE) Vol.4, No.3, July 2015

\section{REFERENCES}

[1] Deborah D.L. Chung, (2010) “Composite Materials Science and Applications”, $2^{\text {nd }}$ edn, Springer, New York

[2] C. Nikhilesh, K.C. Krishan, (2013) "Metal matrix composites", $2^{\text {nd }}$ edn. Springer, New York.

[3] J. Paulo Davim (2012), "Machining of Metal Matrix Composites", Springer, New York

[4] K. U. Kainer (2006), "Metal Matrix Composites. Custom-made Materials for Automotive and Aerospace Engineering”, WILEY-VCH Verlag GmbH \& Co. Weinheim

[5] J.D. Ayers, T.R. Tucker, (1980) "Particulate-TiC-hardened steel surfaces by laser melt injection", Thin Solid Films, 73(1), pp 201-207.

[6] R. Vilar, (2014) "Laser powder deposition", In: Saleem Hashmi ed., Comprehensive Material Processing, Elsevier, USA, pp 163-216.

[7] K.L. Choy, (2003) "Chemical vapour deposition of coatings", Progress in Materials Science, 48, pp 57-170.

[8] K.L. Choy (2000) In: Nalwa HS, ed., "Handbook of nanostructured materials and nanotechnology, vol. 1: synthesis and processing", San Diego (CA): Academic Press.

[9] Donald M. Mattox (2010) "Handbook of Physical Vapor Deposition (PVD) Processing", $2^{\text {nd }}$ edn, Elsevier, USA.

[10] J. A. Hopwood, (2000) In: J A Hopwood ed, "Thin Films: Ionized Physical Vapor Deposition”, Academic Press, San Diego CA, pp 181-207.

[11] Ulf Helmersson, Martina Lattemann, Johan Bohlmark, Arutiun P. Ehiasarian, Jon Tomas Gudmundsson, (2006) "Ionized physical vapor deposition (IPVD): A review of technology and applications", Thin Solid Films, 513, 1-24.

[12] P. Kapranos, C. Carney, A. Pola, M. Jolly (2014) "Advanced Casting Methodologies: Investment Casting, Centrifugal Casting, Squeeze Casting, Metal Spinning, and Batch Casting”, In: Saleem Hashmi ed., Comprehensive Materials Processing, Elsevier, USA, pp 39-67.

[13] J.L. Xu, K.A. Khor, (2011) "Plasma spraying for thermal barrier coatings: processes and applications”, In: H. Xu, H. Guo, eds, Thermal Barrier Coatings, Woodhead Publishing, USA

[14] J. Gandra, H. Krohn, R.M. Miranda, P. Vilaca, L. Quintino, J.F. dos Santos, (2014) "Friction surfacing-A review", Journal of Materials Processing Technology, 214, 1062- 1093.

[15] R.S. Mishra, Z.Y. Ma, (2005) Friction stir welding and processing. Materials Science and Engineering R, 50, pp. 1-78.

[16] R.S. Mishra, M.W. Mahoney, S.X. McFadden, N.A. Mara, A.K. Mukherjee, (2000) High strain rate super plasticity in a friction stir processed $7075 \mathrm{Al}$ alloy, Scripta Materialia. 42, pp. 163-168.

[17] R.S. Mishra, Z.Y. Ma, I. Charit, (2003) "Friction stir processing: a novel technique for fabrication of surface composite", Materials Science and Engineering A, 341:pp 307-310.

[18] B. Ratna Sunil, T.S. Sampath Kumar, Uday Chakkingal, V. Nandakumar, D. Mukesh (2014) Nano-hydroxyapatite reinforced AZ31 magnesium alloy by friction stir processing: a solid state processing for biodegradable metal matrix composites. Journal of Materials Science: Materials in Medicine, 25, pp. 975-988.

\section{Authors}

Dr. B. Ratna Sunil is a graduate and post graduate in Mechanical Engineering and obtained his Ph.D. from Indian Institute of Technology Madras, India (Department of Metallurgical and Materials Engineering). Currently he is working as a faculty in the Department of Mechanical Engineering, Rajiv Gandhi University of Knowledge Technologies, Nuzvid, India. He teaches Metallurgy and Material Science,Manufacturing Processes, Welding Technology and Surface Engineering. He published several papers in peer reviewed international journals and conference proceedings. Presently he is actively involved in doing research in the areas of severe plastic deformation of non-ferrous metals, nanostructured materials; metallic biomaterials and friction stir welding and processing of non-ferrous metals. 